\title{
Effects of Rearing Conditions on the Colonization of Salmonella enteritidis in the Cecum of Chicks
}

\author{
Hiroshi ASAKURA $^{1)}$, Osamu TAJIMA ${ }^{1)}$, Masahisa WATARAI ${ }^{1)}$, Toshikazu SHIRAHATA ${ }^{1)}$, Hisao KURAZONO $^{2)}$ and \\ Sou-ichi MAKINO')*
}

${ }^{1)}$ Department of Veterinary Microbiology, Obihiro University of Agriculture and Veterinary Medicine, Hokkaido 080-8555 and

${ }^{2)}$ Department of Medical Technology, School of Health Sciences, Okayama University, Okayama 700-8558, Japan

(Received 31 March 2001/Accepted 2 July 2001)

ABSTRACT. Salmonella enteritidis is the cause of human salmonellosis associated with contaminated eggs. In this study, we artificially challenged $S$. enteritidis to chicks just after hatching, and the effects of breeding conditions on the intestinal carriage of $S$. enteritidis were examined. S. enteritidis was not directly detected from spleen, liver and blood, but were constantly isolated from the cecal contents throughout the experiment. When chicks were reared in the unsanitary conditions and in the high housing density, the numbers of $S$. enteritidis increased. The subsequent experiment was undertaken to examine whether the antibacterial additive in a feed would have any impact on S. enteritidis colonization in chicks. Some antibiotic effective on the growth promotion had an influence on $S$. enteritidis colonization.

KEY WORDS: cecum, chick, Salmonella enteritidis.

J. Vet. Med. Sci. 63(11): 1221-1224, 2001

Food-born disease caused by Salmonella, especially by hen eggs and processed eggs contaminated with $S$. enteritidis, has been increasing [2, 7, 12]. Although young chicks are highly sensitive to $S$. enteritidis [8], salmonellosis has not taken noticed as an infectious disease in adult chicks because of its low disposed rate and latent symptom [6]. However, chicken eggs contaminated with $S$. enteritidis had become a big social problem with increasing the occurrence of the food-born disease by Salmonella [11]. Excretion of $S$. enteritidis in feces is the main factor for the contamination. In this study, we artificially inoculated $S$. enteritidis into chicks to examine the effects of environmental and management factors such as sanitation and housing density on the intestinal carriage of $S$. enteritidis in chick.

Tetracycline-resistant $S$. enteritidis strain No.273 isolated from a patient with salmonellosis was grown in L-broth at $37^{\circ} \mathrm{C}$ for $16 \mathrm{hr}$, and its aliquot was artificially inoculated into the crops of White-Leghorn chicks within $24 \mathrm{hr}$ after hatching through the oral catheter. The standard experimental diets for poultry, SDL No. 1 and No. 4 (Nippon Formula Feed Co. Ltd., Aichi, Japan) without any antibiotics were used as the feeds for chicks through the experiments. To rear chicks, two types of cages, sanitary and unsanitary cages, which were 70 by 50 by $50 \mathrm{~cm}$ and had the base of 0.5 $\mathrm{cm}$ square mesh, were prepared; in sanitary cage, the base was exchanged to a new one in the morning and the evening twice a day, but, in unsanitary cage, the base was fixed through the experiments.

S. enteritidis cells $\left(8.0 \times 10^{4}\right)$ were inoculated into 25 chicks, and then divided into 5 sanitary cages of 5 chicks each. Spleens, livers, blood and cecum were carefully iso-

\footnotetext{
* Correspondence to: Dr. Makino, S., Department of Veterinary Microbiology, Obihiro University of Agriculture and Veterinary Medicine, Inada-cho, Obihiro, Hokkaido 080-8555, Japan.
}

lated from all chicks in each cage at 2, 5, 6 and 7 weeks post infection (wpi), were weighed and homogenized in $1 \mathrm{~m} l$ of PBS. Aliquots of the suspensions were directly spread on DHL agar (Eiken Co., Ltd., Tokyo, Japan) plates supplemented with $10 \mu \mathrm{g} / \mathrm{m} l$ of tetracycline to measure the colony forming units (CFU), resulting that average $2.6 \times 10^{6} \mathrm{~S}$. enteritidis cells were detected from cecal contents without any symptoms but not from other samples. Then, each 0.1 $\mathrm{m} l$ of those suspensions was incubated in $10 \mathrm{ml}$ of Hajna Tetrathionate broth (HTB; Eiken Co., Ltd., Tokyo, Japan ) at $37^{\circ} \mathrm{C}$ for $20 \mathrm{hr}$, followed by spreading on DHL agar plates with tetracycline, resulting that $S$. enteritidis cells were detected in the enrichment cultures of liver and spleen (data not shown). The remaining 5 chicks in a cage were separately reared in 5 sanitary cages at $7 \mathrm{wpi}$, and then, the fresh cecal droppings were collected to measure the CFU weekly for up to $37 \mathrm{wpi}$, but average $2.8 \times 10^{6}$ cells were constantly detected for up to 37 wpi without any symptoms. In addition, the rapid clearance of other bacterial species as Escherichia coli and Staphylococcus aureus from the intestinal contents was observed at $2 \mathrm{wpi}$ (data not shown). That is to say, since the chick, which had been once infected with $S$. enteritidis, would become a carrier of Salmonella, colonization of Salmonella in the chicks should become a serious problem on the food hygiene.

$S$. enteritidis cells $\left(7.0 \times 10^{4}\right)$ were inoculated into 120 chicks, which were divided in four flocks; 4 sanitary cages of 5 chicks, 4 sanitary cages of 10 chicks, 4 unsanitary cages of 5 chicks and 4 unsanitary cages of 10 chicks. The CFU of $S$. enteritidis in the ceca of all chicks in each group were measured weekly for up to 3 wpi. As the results, the CFU of the samples from the sanitary cages were always lower than those from the unsanitary cages, and also the CFU of the samples at rearing 5 chicks in a cage were always lower than 


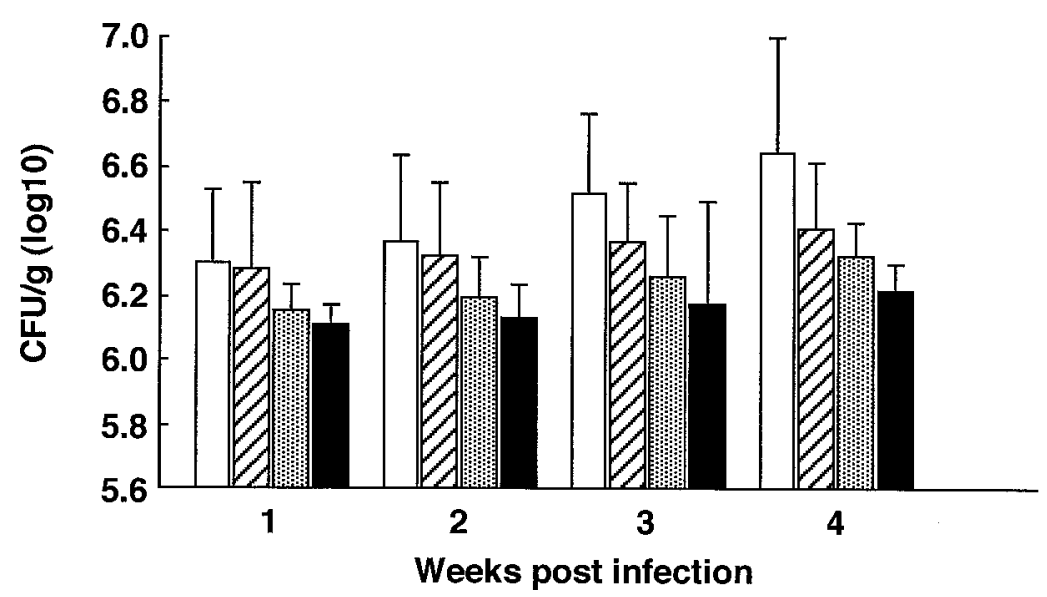

Fig. 1. Influences of the breeding conditions on the carriage of $S$. enteritidis in the cecal contents. Total 120 chicks were divided in four flocks; 4 unsanitary cages of 10 chicks $(\square), 4$ sanitary cages of 10 chicks $(\square), 4$ unsanitary cages of 5 chicks (再) and 4 sanitary cages of 5 chicks

those at rearing 10 chicks in a cage (Fig. 1). Those results show that the environmental and management factors, such as sanitation and housing density, influenced on the colonization of $S$. enteritidis in the cecum. That is to say, when chicks were reared in the unsanitary conditions and in the high housing density, the numbers of $S$. enteritidis in the cecum increased. Such stresses would encourage the Salmonella colonization in the cecum.

Uninfected chicks within $24 \mathrm{hr}$ after hatching were newly introduced in the empty cages that were not disinfected after all infected chicks were removed, and the CFU in the cecal contents of the newly introduced chicks were measured. After 1 week from the introduction, $S$. enteritidis was colonized in cecal contents in all chicks (data not shown). This colonization would be caused by picking up contaminated feces from infected chicks in the former experiment. Especially, in unsanitary cages, since chicks could easily contact with contaminated feces, $S$. enteritidis cells were continuously inoculated into chicks and their colonizations in the cecum would be encouraged. Generally, it takes 2 to 4 weeks to form normal bacterial intestinal flora in chicks. However, chicks just after hatching are separated from adult birds in modern chick rearing, and their intestinal normalflora is formed naturally of various kinds of bacterial taken from the environments. Competitive exclusion, which prevents $S$. enteritidis colonization by rapidly establishing normal intestinal bacterial flora by adding intestinal bacterial flora of adult birds to chicks, has been considered [7]. This technology may be useful to remove efficiently $S$. enteritidis from the cecal contents of chicks.

Generally, newly hatched chicks were highly sensitive to $S$. enteritidis and usually died with only one $S$. enteritidis cell $[6,8]$. Moreover, the long-term excretion of $S$. enteritidis into feces as shown in this study were rarely reported [8]. However, we showed in this study that, when about $10^{4} \mathrm{~S}$. enteritidis cells were infected into chicks within $24 \mathrm{hr}$ after hatching, S. enteritidis cells were constantly excreted in cecal droppings without any symptoms for 37 wpi but isolated from spleen and liver only by the enrichment culture. S. enteritidis exhibiting strong pathogenicity to chick was mostly phage type $4[1,3-5,9,13]$, but other phage type $S$. enteritidis like phage type 8 exhibited no symptoms to chicks [10]. Although why S. enteritidis strain No. 273 used in this study was non-pathogenic to chicks and colonized in the cecum was not clear, this strain might be avirulent to chicks although it was derived human infection and might not be phage type 4. Anyway, if once even $S$. enteritidis cells avirulent to chicks were inoculated in young chicks, they would form normal bacterial intestinal flora, be excrete in the feces for a long time and finally a lot of hens would be carriers of $S$. enteritidis.

We examined the long-term colonization of $S$. enteritidis cells in chicks, but antibiotics are usually used as feed additives for chicks just after hatching for the purpose of their growth promotion and preventing infectious disease. However, their influences on the intestinal carriage of $S$. enteritidis in chick were not examined in detail. Therefore, eight kinds of agents, avilamycin (AVM), enramycin (ER), efrotomycin (EFM), lasalocid (LS), monensin (MN), nosiheptide (NHT), salinomycin (SLM), and virginiamycin (VGM), were mixed with SDL No. 1 at the final concentrations of $10,5,6,75,80,5,50$ and $75 \mathrm{ppm}$, respectively. Those concentrations are ordinary used for the growth promotion of chicks. Feed with each antibiotic was given to 20 chicks within $24 \mathrm{hr}$ after hatching, which were divided into 4 sanitary cages of 5 chicks each, and $9.0 \times 10^{4}$ cells of $S$. enteritidis were inoculated orally in each chick. The CFU of $S$. enteritidis in the cecal contents of all chicks in each cage were measured weekly for up to 3 wpi. When ER, NHT, MN, SLM and LS were added to the feed, the CFU of $S$. enteritidis in the cecum were mostly identical with the control (Fig. 2). However, when AVM, NHT and EFM were 


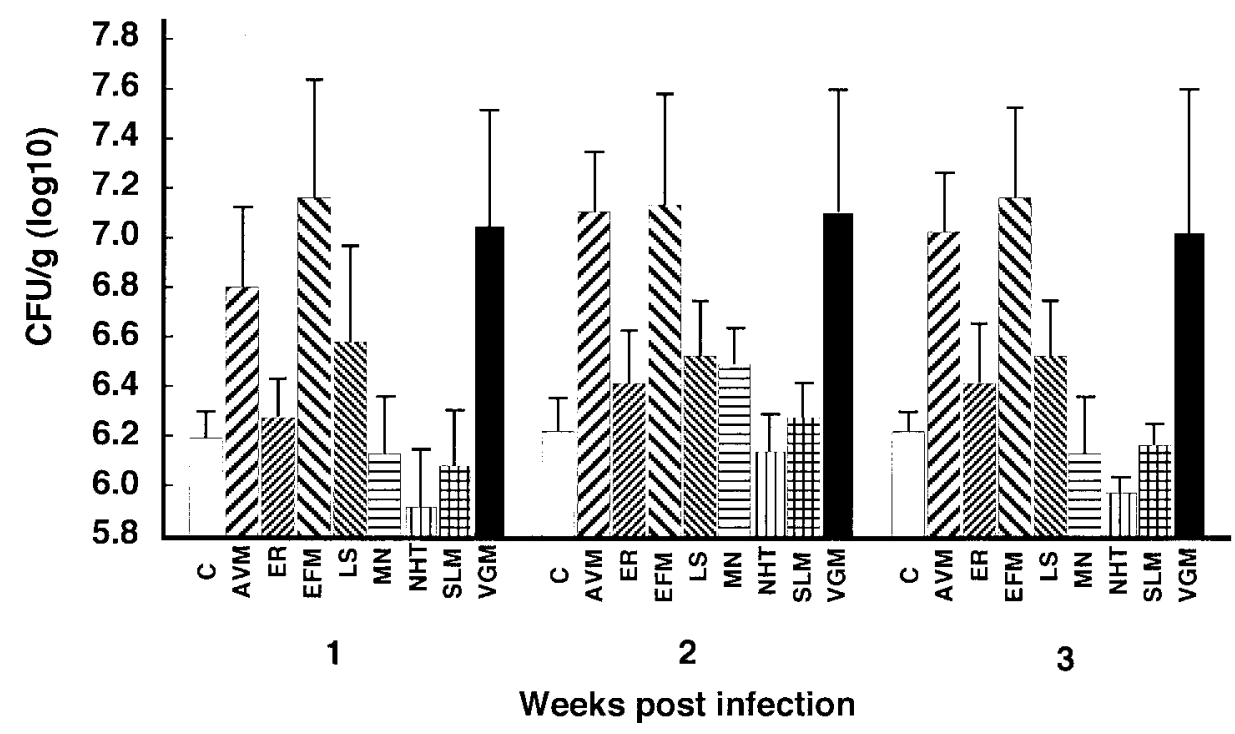

Fig. 2. Influences of antimicrobial agents on the carriage of S. enteritidis in the cecal contents. Eight kinds of agents, avilamycin (AVM), enramycin (ER), efrotomycin (EFM), lasalocid (LS), monensin (MN), nosiheptide (NHT), salinomycin (SLM), and virginiamycin (VGM), were mixed with the feed. C, the feed without antibiotics as the control.

added to the feed, the CFU of $S$. enteritidis were greater than the control and feed with other antibiotics (Fig. 3). MN, SLM and LS, which are anticoccidial drugs, did not affect $S$. enteritidis for colonization, because they prevent sporozoite from penetration the intestine at the initial stage of coccidial infection. On the other hand, AVM, VGM and EFM agents increased S. enteritidis colonization (Fig. 2). These antibiotics, which are antimicrobial activities mainly against gram-positive bacteria, reduced the number of gram-positive enteric bacteria, resulting in forming a suitable intestinal environment for $S$. enteritidis colonization. However, ER and NHT, which were also antimicrobial antibiotics against gram-positive bacteria, did not increase $S$. enteritidis colonization in the chicks. Since both ER and NHT have been used for over 20 years, the sensitivities of gram-positive bacteria in the intestine of chicks to both drugs might have been decreased. From the results, it is clear that some antimicrobial agents increased $S$. enteritidis colonization in the intestine.

Next, 40 chicks within $24 \mathrm{hr}$ after hatching were divided in two flocks of 20 chicks, and 5 chicks each were reared in a sanitary cage. To 20 chicks, feed without antibiotics was given for 5 weeks. To the remaining 20 chicks, feed with AVM (10 ppm) was given for the first 3 weeks, and feed without antibiotics was given for the additional 2 weeks. Then, $7.5 \times 10^{4}$ cells of $S$. enteritidis were inoculated orally in each chicks, and the CFU of the cecal contents of all chicks were measured weekly for up to 5 wpi. When the feed with antibiotics was given, the CFU were greater than that in the case without antibiotics (Figs. 2 and 3). However, when feed without antibiotics was given at 3 wpi, the CFU decreased drastically (Fig. 3). Limiting their practical appli-

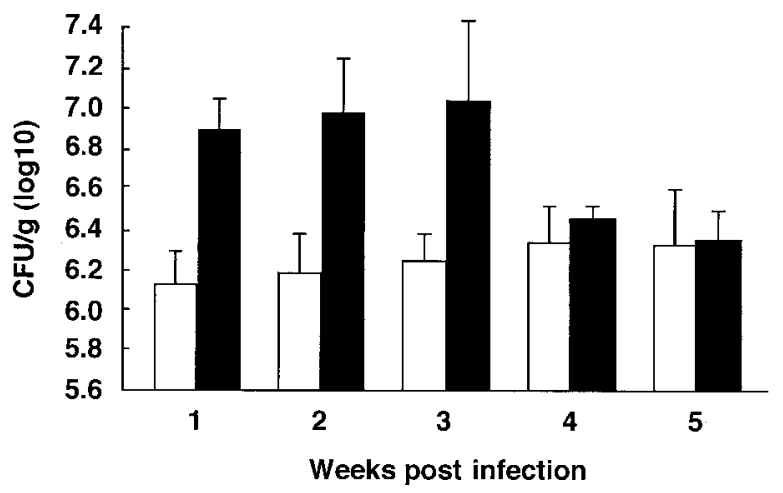

Fig. 3. Influences of antimicrobial agents on the carriage of $S$. enteritidis in the ceca. Feeds with AVM were given during first 3 weeks, and then antibiotic-free feeds were given during the next 2 weeks $(\boldsymbol{\square})$. As the control, feeds without antibiotics were given to chicks $(\square)$.

cations immediately is difficult, even though they allow $S$. enteritidis colonization because they have a big advantage to promote growth of chicks.

ACKNOWLEDGMENTS. This work was supported in part by grants from the Ministry of Health, Labour and Welfare (Research on Emerging and Re-emerging Infectious Diseases). H. Asakura is a research fellow of Japan Society for the Promotion of Science (JSPS).

\section{REFERENCES}

1. Cooper, G. L., Nicholas, R. and Bracewell, C. D. 1989. Vet. 
Rec. 125: 567-572.

2. Gast, R. K. and Beard, C. W. 1993. J. Food Prot. 56: 21-24.

3. Hinton, M., Threlfall, E. J. and Rowe, B. 1991. FEMS Microbiol. Lett. 70: 193-196.

4. Hopper, S. A. and Mawer, S. 1989. Vet. Rec. 123: 351.

5. Lister, S. A. 1988. Vet. Rec. 122: 214.

6. Morris, G. K., McMurray, B. L., Galton, M. M. and Wells, J. G. 1969. Am. J. Vet. Res. 30: 1413-1421.

7. Nisbet, D. J., Tellez, G. I., Lowry, V. K., Anderson, R. C., Garcia, G., Nava, G., Kogut, M. H., Corrier, D. E. and Stanker, L. H. 1998. Avian. Dis. 42: 651- 656.

8. Nakamura, M., Nagamine, N., Suzuki, S., Norimatsu, M.,
Oishi, K., Kijima M., Tamura, Y. and Sato, S. 1993. J. Vet. Med. Sci. 55: 694-653.

9. O'Brien, D. P. 1988. Vet. Rec. 122: 214.

10. Shivaprasads, A. D., Timony, J. F., Molales, S., Luis, B. and Baker, R. 1990. Avian. Dis. 43: 548-557.

11. Sin, J., Quigley, C. and Davies, M. 2000. Dis. Public Health 3: 90-94.

12. Trepka, M. J., Archer, J. R., Altekruse, S. F., Proctor, M. E. and Davis, J. 1999. J. Infect. Dis. 180: 1214-1219.

13. Timmony, J. F., Shivaplasad, H. L., Baker, R. C. and Rowe, B. 1989. Vet. Rec. 125: 600-601. 